\title{
A Comparison of Neuronal Growth Cone and Cell Body Membrane: Electrophysiological and Ultrastructural Properties
}

\author{
Peter B. Guthrie, ${ }^{1}$ Robert E. Lee, ${ }^{2}$ and Stanley B. Kater ${ }^{1}$ \\ ${ }^{1}$ Program in Neuronal Growth and Development and ${ }^{2}$ Department of Anatomy and Neurobiology, Colorado State \\ University, Ft. Collins, Colorado 80523
}

\begin{abstract}
This study investigated a broad set of general electrophysiological and ultrastructural features of growth cone and cell body membrane of individual neurons where membrane from different regions of the same neuron can be directly compared. Growth cones were surgically isolated from identified adult Helisoma neurons in culture and compared with the cell body using whole-cell patch-clamp recording techniques. All isolated growth cones generated overshooting regenerative action potentials. Five neurons (buccal neurons B4, B5, and B19; pedal neurons P1 and P5) were selected that displayed distinctive action potential waveforms. In all cases, the growth cone action potential was indistinguishable from the cell body action potential and different from growth cones from other identified neurons. Two of these neurons (B5 and B19) were studied further using voltageclamp procedures; growth cones and cell bodies again revealed major similarities within one neuron type and differences between neuron types. The only suggested difference between the growth cone and cell body was an apparent reduction in the magnitude of the A-current in the growth cone. Peak inward and outward current densities, as with other electrophysiological features, were different between neuron types, but were, again, similar between the growth cone and the cell body of the same neuron. Freeze-fracture analysis of intramembraneous particles (IMPs) was also performed on identified regions of the same neuron in culture. Both the density and the size distribution of IMPs were the same in growth cone, cell body, and neurite membranes. In these general electrophysiological and ultrastructural characteristics, therefore, growth cone membranes appear to retain the identity of the parent neuron cell body membrane.
\end{abstract}

The unique motile and decision-making properties of the growth cone might suggest that the membrane of the growth cone is markedly different from membrane of other regions of the neuron. Growth cones serve many unique roles during the development of the nervous system. Activity of the growth cone, in response to both intrinsic and environmental signals, determines whether it will advance, branch, turn, follow, or synapse with one of the potential targets within its immediate vicinity. Decisions made by the growth cone determine a neuron's final

\footnotetext{
Received June 16, 1988; revised Feb. 27, 1989; accepted Mar. 2, 1989.

We would like to thank Drs. M. Bunge, K. Pfenninger, and D. Landis for critical comment on earlier versions of the manuscript, and M. Mischke and D. Dehnbostel for technical assistance. Supported by NS24683.

Correspondence should be addressed to Peter B. Guthrie at the above address. Copyright (C) 1989 Society for Neuroscience $0270-6474 / 89 / 103596-10 \$ 02.00 / 0$
}

form and connectivity patterns. The ability of the growth cone to make such decisions, even in the absence of communication with its cell body, has been shown in a number of studies (Gundersen and Barrett, 1980; Haydon et al., 1987).

Several studies have shown differences between the general membrane properties of growth cones and the general membrane properties of the rest of the neuron. For example, differences have been seen in binding of spccific lcctins to growth cone membrane compared with membrane from other regions of neurons (Pfenninger and Maylie-Pfenninger, 1981; Lankford and Klein, 1985). The unique properties of the growth cone might also be due to differences in intracellular components. For example, the membrane-associated phosphoprotein GAP43 is localized in growth cones (Meiri et al., 1986; Skene et al., 1986). Finally, significant differences between axon and growth cone in the density of intramembrane particles (IMPs) have been seen (Pfenninger and Bunge, 1974; Small, 1985), suggesting that the growth cone may not possess all of the membrane properties associated with electrically active axonal membrane.

Many neurons, however, possess unique identities. Proper identification of individual neurons is critical for formation of the nervous system (Raper et al., 1983). In addition, some of the distinctive properties of individual neurons probably contribute to proper function of those neurons. One would therefore expect to see some neuron-selective properties shared throughout a single neuron. For example, 2 specific ion channels have previously been found both in growth cones and cell bodies of individual neurons. A serotonin-modulated potassium channel which underlies sensitization (Klein et al., 1982; Siegelbaum et al., 1982) was initially found on the cell body of Aplysia neurons; with the refinement of ccll culturc procedures for these neurons, this channel has now also been found on growth cones (Belardetti et al., 1986a). A cation-selective inward channel has also been found in both growth cone and cell body membrane in Helisoma neurons (Cohan et al., 1985). In addition, the synchronous masking of this channel in both the growth cone and cell body suggests that the channel is similarly regulated in both locations. In view of these membrane similarities in structures with markedly different functions, we decided to undertake a broader characterization of membrane properties to determine whether the growth cone membrane is distinct in its general properties from other regions of the same neuron.

Identified Helisoma neurons with previously characterized properties have proven useful in determining environmental signals controlling the growth status of neurons (Haydon et al., 1985; Cohan and Kater, 1986; Haydon et al., 1987; Kater and Mattson, 1987); these cultured neurons now provide us with the opportunity to study the same specified regions of individual 
identified neurons with both electrophysiological and ultrastructural techniques. The results described below suggest that the membrane of the growth cone is quite similar, in its general electrophysiological and ultrastructural properties, to membrane from the cell body.

A preliminary report of this work has appeared (Guthrie et al., 1986).

\section{Materials and Methods}

Individual identified neurons were isolated from adult Helisoma nervous system and cultured as previously described (Kater and Mattson, 1987). Five different neurons were used in the course of this study: buccal ncurons B4, B5, and B19, and pedal neurons P1 and P5. Within 12-24 hr after plating, Helisoma neurons start a phase of active outgrowth that will typically last $4-5 \mathrm{~d}$. All of the data in this report are taken from neurons during this period of active outgrowth.

Electrophysiological studies. Standard patch-electrode recording procedures were used to characterize action potential waveforms and the ionic currents in both cell bodies and in isolated growth cones at room temperature. Electrodes were fabricated on a Narishigi PP-83 patch pipette puller from borosilicate glass (1.5 mm O.D.; F. Haer and Co.). The intracellular patch solution contained $55 \mathrm{~mm} \mathrm{~K}$-aspartate, $2 \mathrm{mM}$ EGTA, 2 mм $\mathrm{MgCl}_{2}$, 5 mм glucose, and 5 mм HEPES, pH 7.4, 125 $\mathrm{mmol} / \mathrm{kg}$ osmolality. Electrode resistances for cell body recording were 3-5 M 2 ; for isolated growth cones, 15-20 M $\Omega$ electrodes were used. A Dagan 8900 patch amplifier with a $0.1 \mathrm{G} \Omega$ headstage was controlled by an Indcc LSI 11/23-based computer system, which was also used to collect and analyze the data.

Growth cones were isolated from the rest of the neuron by severing the neurite immediately proximal to the growth cone, using a glass electrode as a microknife (see Fig. 1). Voltage-clamp records from isolated growth cones (e.g., Fig. 4) demonstrate that these preparations were electrically compact and easily space-clamped; in addition, the membrane properties of such isolated growth cones could be studied with minimal contamination from (possibly different) neurite membrane. All recordings were made within $30 \mathrm{~min}$ of isolation of the growth cone. Cell body recordings were often made from the same neuron used for isolated growth cone preparations. Voltage-clamp analyses were only made on neurons from which all neurites had been "pruned"; preliminary studies indicated that the action potential waveform was not significantly altercd by this procedure, while voltage-clamp records showed this "pruning" to be necessary for adequate space clamp of inward currents. Action potential waveforms could be obtained from intact neurons.

The isolation of the growth cone raises the question of what delineates the growth cone proper. The neurite ending is composed of 2 very discrete regions: The growth cone is, by way of analogy, the hand (a region extending proximally as far as the surrounding veil), and the neurite is the arm. Between them, however, is the less discrete region of the "wrist," a region of unspecified designation as either neurite or growth cone. For this study, isolation was performed at the region of the wrist to minimize electrical contribution from neurite membrane (see Fig. 1). In order to avoid interpretational problems, we here define the growth cone as the most terminal region of the neurite including a transition zone to "mature" neurite not to exceed $100 \%$ of the length of the growth cone. We estimate, from membrane capacitance measurements, that the transition zone of the neurite would, under the worst conditions (a length of neurite as long as the growth cone), make a very small contribution $(<17 \%)$ to the total surface membrane seen in growth cone recordings.

All action potentials analyzed for this study were evoked with depolarizing current pulses (100 msec duration, minimum current necessary to evoke an action potential during the stimulus) from resting membrane potential (ca. $-60 \mathrm{mV}$ ) at intervals of not less than $30 \mathrm{sec}$ to avoid spike broadening. Action potential waveforms were sampled at $10 \mathrm{kHz}$. The maximum amplitude of the action potential was defined as the difference between the most positive and the most negative voltages of the waveform. The width at half-amplitude (half-width) was determined at the voltage halfway between those extremes. The amplitude and the half-width were determined from 3-6 action potentials for each recording; the mean of these determinations was used for subsequent analysis.

For voltage-clamp records, the preparations were held at $-50 \mathrm{mV}$;
$100 \mathrm{mscc}$ duration command stcps starting at $60 \mathrm{mV}$ and incrementing by $10 \mathrm{mV}$ to $+50 \mathrm{mV}$ were given every $2 \mathrm{sec}$. Leakage and capacitative currents were subtracted. The capacitative current was integrated to estimate the membrane area under voltage clamp, assuming $1 \mu \mathrm{F} / \mathrm{cm}^{2}$ (Rall, 1977). Maximum peak inward and outward currents were normalized against this measure of membrane surface area to estimate membrane current densities.

Freeze-fracture electron microscopy. Neurons were grown on glass coverslips and fixed with $3 \%$ glutaraldehyde in $0.1 \mathrm{~m}$ phosphate buffer $\left(\mathrm{pH} \mathrm{7.2)}\right.$ for $1 \mathrm{hr}$ at $4^{\circ} \mathrm{C}$. The coverslips were washed twice in $0.1 \mathrm{M}$ phosphate buffer. The material was cryoprotected by infiltration of $25 \%$ glycerol in $0.1 \mathrm{M}$ phosphate buffer at $4^{\circ} \mathrm{C}$ for $30 \mathrm{~min}$. Pieces of glass containing the neurons were obtained by scoring and breaking the coverslip. The neurons were frozen according to the method of Pauli et al. (1977), which consists of placing a drop of a solution containing polyvinyl alcohol, phosphate-buffer saline, glycerol, and dimethylsulfoxide onto a 3-mm-diameter gold specimen support with a central $1 \mathrm{~mm}$ hole. The glycerol solution was drained from a piece of glass with an attached neuron, and the glass was placed neuron-side down onto the polyvinyl alcohol solution drop on the specimen support. The specimen was then frozen by plunging the gold support into partially liquid Freon-12 cooled in liquid nitrogen.

The frozen neurons on glass were fractured in a Balzers 301 at $-100^{\circ} \mathrm{C}$ and $10^{-6} \mathrm{mbar}$ by placing the cold knife under the glass and raising the knife. The glass popped off with the fracture plane typically passing through the neuron. Platinum-carbon was immediately deposited on the fractured surface at an angle of $40^{\circ}$. Carbon was evaporated from above to strengthen the replicas. The specimen was dissolved in fullstrength bleach for $4 \mathrm{hr}$, the replicas washed in water and picked up on grids. The replicas were viewed in a Philips $400 \mathrm{~T}$ transmission microscope. IMPs were counted in selected 1- $\mu \mathrm{m}$-square regions from photographic prints at a nominal magnification of $100,000 \times$. IMP diameters (perpendicular to the direction of the shadow) were measured from the same prints using a micrometer loupe.

\section{Results}

\section{Electrophysiological responses of the growth cone and the} soma

Individual isolated Helisoma neurons generate significant outgrowth when placed into culture (Fig. 1A). Growth cones were easily severed from the cell body using a glass electrode as a microknife (Fig. $1 B$ ). Such isolated growth cones remain motile and will continue to elongate and generate normal resting membrane potentials and action potentials for over $3 \mathrm{~d}$ after isolation. Whole-cell patch-recording configurations were readily obtained from both the cell bodies and the growth cones. All healthy growth cones responded to depolarizing current injection with an overshooting, regenerative action potential (Fig. 2).

Recordings from isolated growth cones show that growth cone action potentials are identifiable to the same extent as the cell bodies are. Previous studies (Hadley et al., 1982; Cohan et al., 1985) have shown that individual Helisoma neurons can be identified by their characteristic action potential waveforms. In fact, the action potential waveform often serves as a "fingerprint," unique to each neuron type. The top portion of Figure 2 shows stimulated action potential records from $4 \mathrm{~B} 19$ growth cones (top left), all from the same parent B19 neuron (bottom left), and 3 B5 growth cones (top right), all from the same parent B5 neuron (bottom right). The action potentials from both the B19 cell body and growth cones are significantly faster than the action potentials from the B5 cell body and growth cones.

In Figure 3, the width of the action potential at half-amplitude (half-width) was determined for cell bodies and growth cones from 5 different identified neuron types from the buccal and pedal ganglia. In each case, the half-width of the cell body action potential proved to be an excellent predictor of the half-width 



Figure 1. Isolation of growth cones and cell bodies. A, Phase-contrast image of a Helisoma neuron B5 before isolation of the growth cones. Scale bar, $25 \mu \mathrm{m}$. $B$, Higher-magnification view of the neurite and growth cone outlined in $A$. The scissors indicate the sites of transection. $C$, The growth cone has been surgically isolated from the neurite using a patch electrode as a microknife. The neurite was then severed from the cell body to remove the electrical load of neurite membrane, improving space-clamp of the cell body. Scale bar, $25 \mu \mathrm{m}$.

of the growth cone action potential (coefficient of correlation = 0.980 ). This correspondence suggests that the membrane of the growth cone and the cell body contain similar relative densities of the ionic channels contributing to the action potential.

\section{Voltage-clamp recordings}

The action potential waveform is only a general indicator of membrane properties. Voltage-clamp techniques provide a more detailed comparison of the kinetics of the general underlying ionic currents. Figure 4 shows characteristic records from an isolated neuron B19 growth cone (top left) and an isolated neuron B5 growth cone (top right), with voltage clamp records of the cell bodies shown below. Different identified neurons showed different characteristic voltage-clamp currents, with the neuron B19 having a faster and larger inward current. Although no attempt was made to pharmacologically dissect individual currents, a consistent difference between the growth cone and cell body voltage-clamp records was the greatly reduced A-current (Connor and Stevens, 1971) in the growth cones. In many growth cones, evidence for an A-current could only be seen when the holding potential was reduced to $-100 \mathrm{mV}$. In all other respects, the time courses of the voltage-clamp currents in the cell body and the growth cones were similar within a neuron type; a comparison of the 5 different identified neurons suggested that the growth cone voltage-clamp currents were more similar to their parent cell body than to growth cones from another neuron type.

The correspondence of the action potential durations in the growth cone and cell body membranes could be due either to similar relative or to similar absolute, densities of ion channels. The membrane surface area under voltage clamp was estimated by integrating the capacitative current (see Materials and Methods). Peak inward and outward currents from buccal neurons B5 and B19 were normalized to the membrane surface area to estimate the current density through a unit patch of membrane. Both growth cone and cell body membranes showed similar absolute densities of the peak inward and outward currents (Fig. 5 ). Unpaired $t$ test analysis of the data confirmed that the cell body and growth cone current densities were not statistically different $(p>0.15)$, whereas the current densities for B5s and B19s were statistically different $(p<0.001)$. Again, the growth cone current densities were typical for the neuron type and not the neuron region.

\section{Ultrastructural comparison of growth cone and soma membrane}

Both the action potential and the voltage-clamp analyses demonstrated that the functional properties of the growth cone membrane are remarkably similar to the soma membrane. Previous studies in other species, however, have suggested that growth cone membrane, unlike cell body membrane, is greatly dimin- 



B5 CELL BODY

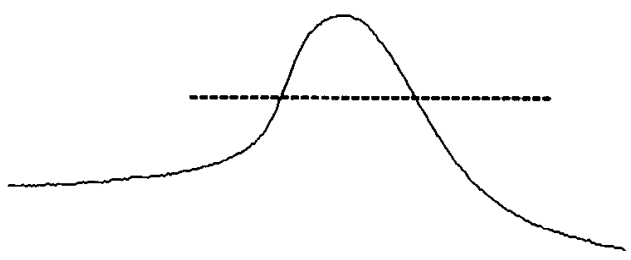

Figure 2. Growth cones and the cell bodies have similar action potentials. Action potentials were evoked from rest potential with $100 \mathrm{mscc}$ depolarizing current pulses. The top traces are superimposed from 4 (B19) or 3 (B5) growth cones; the growth cones were isolated from the cell bodies shown in the lower traces. Action potentials in B19 cell bodies and growth cones consistently appeared faster than action potentials in B5 cell bodies and growth cones. The action potentials are displayed on an expanded time scale to emphasize the difference in time courses; the most negative voltage used to define action potential amplitude in Figure 3 is therefore not visiblc. Dotted lines indicate zero potential. ished in IMPs (Pfenninger and Bunge, 1974; Small, 1985). Since this observation was not consistent with our studies of active membrane properties in isolated growth cones, we also examined freeze-fracture images of Helisoma neurons. A more general description of membrane characteristics can be provided by freeze-fracture analysis of the density and size distribution of IMPs; while freeze-fracture analysis cannot determine the functional status of the various proteins the IMPs represent, it does give a general overview of the organization of membranes.

One of the major advantages of the Helisoma system is the ability to locate, within the freeze-fracture replica, identified regions of single neurons. Individual identified neurons were grown on glass coverslips, fixed, and prepared for freeze-fracture analysis in the transmission electron microscope. For this procedure, growth cones were not isolated from the cell body. As shown in Figure 6, one can easily proceed from a phase-contrast photograph of the neuron to the freeze-fracture replica and identify not only regions of the cell body, but specific regions of neurites, growth cones, and even filopodia. Figure 7 shows a high-magnification image of the 2 fasciculated neurites shown in Figure 6. Figure 8 shows a similar high-magnification image of one of the growth cones shown in Figure 6. It is therefore possible to examine the density and size distribution of IMPs within discrete, identified regions of a single neuron.

Both neurons B19 and B5 were examined by freeze-fracture techniques. One micrometer squares were selected from the growth cone, neurite, and cell body, and the number and size of IMPs within each square were determined. Figure $9 A$ shows that the overall density of IMPs was also very similar in all regions of neurons B19. Figure $9 B$ shows that the size distributions of IMPs of neurons B19 were similar in the growth cones, neurites, and cell bodies. Indistinguishable results were obtained with neurons B5 (data not shown).

All the measurements reported here are from the surface apposed to the glass coverslip and represent P-face membrane. Previous freeze-fracture of some invertebrate neurons have shown gap-junction particles to partition into the E-face, while, in vertebrates, gap junction particles partition into the P-face (Peracchia, 1973). It is therefore possible that particles in the E-face might show differences between the growth cone and the cell body. Occasionally (ca. 10\%), however, the fracture plane would pass through the upper membrane exposed to the medium (E-face membrane); similar IMP densities and size distributions were found in those replicas. Ideally, replicas of the E-face apposed to the glass and of P-face exposed to the medium should also be examined. This would require replication of the

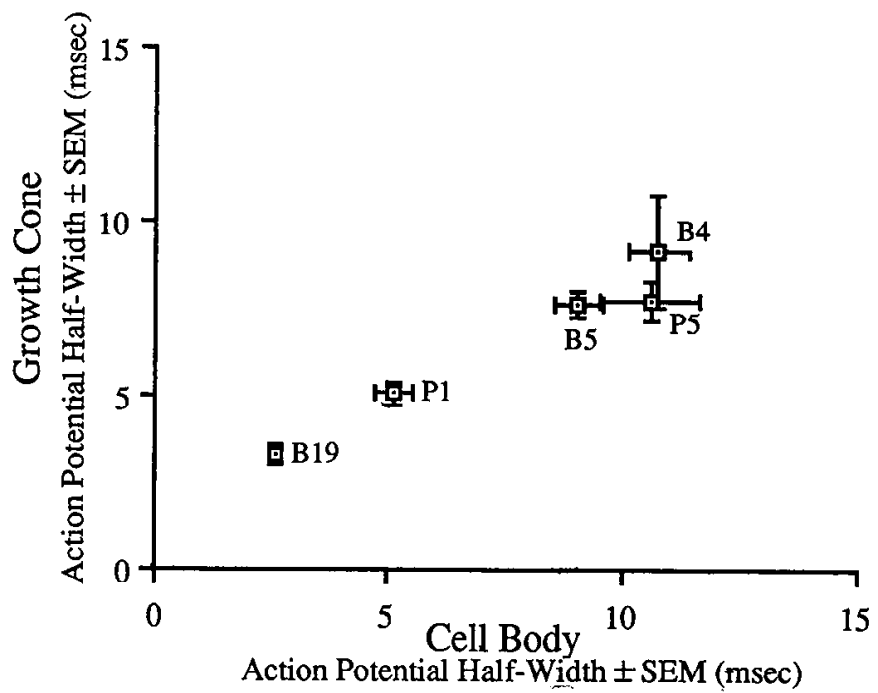

Figure 3. Growth cones and the cell bodies have similar characteristic identifying action potential waveforms. Action potentials were stimulated in isolated cell bodies and isolated growth cones of 5 different identified neurons. The amplitude was defined as the difference between the most positive and the most negative voltages of the waveform. The width at half-amplitude (half-width) was determined at the voltage halfway between those extremes. The values represent average \pm SEM for 5-42 growth cones and 4-63 cell bodies. The coefficient of correlation for the averages was 0.980 . 
Figure 4. Growth cones and the cell bodies have similar characteristic identifying voltage-clamp waveforms. Representative whole cell/whole growth cone voltage-clamp traces from growth cones (top) and cell bodies (bottom) are shown for neurons B19 (right) and B5 (left). The holding voltage was $-50 \mathrm{mV}$; voltage steps (100 msec duration) to $-30,-10,10,30$, and $50 \mathrm{mV}$ are shown (marked at left of the B5 growth cone record). Leakage and capacitative currents have been subtracted. Inward currents in B19's were consistently faster than the inward currents in B5's. The ratio of inward to outward currents was also much larger for $\mathrm{B} 19$ cell bodies and growth cones (1.01 and 1.17, respectively) than for $B 5$ cell bodies and growth cones ( 0.55 and 0.56 , respectively) (see also Fig. 5). The only consistent difference observed between cell bodies and growth cones was the marked reduction of an A-current in the growth cones. The "hump" in the cell body current traces (arrowheads) is typical of the A-current that has been pharmacologically characterized in other preparations by other investigators.
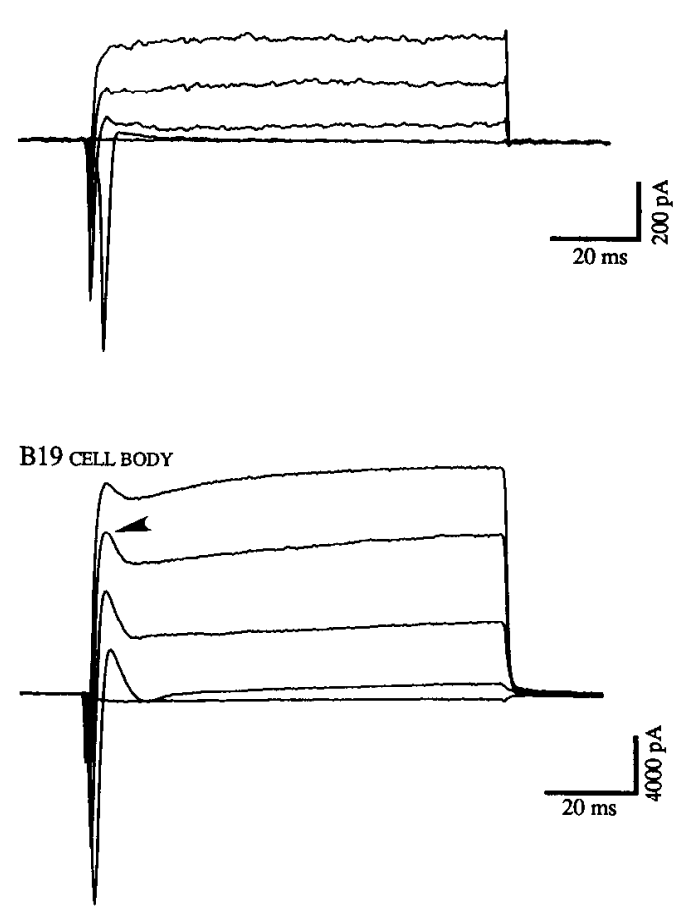

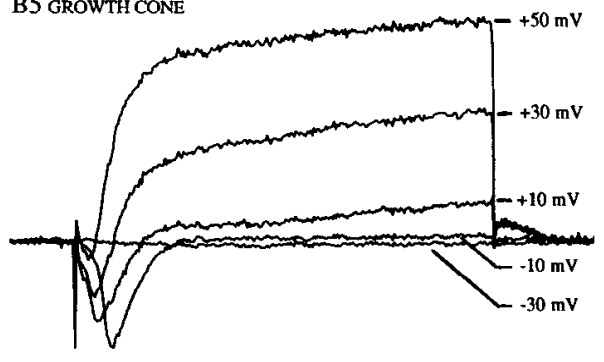

B5 CELL BODY

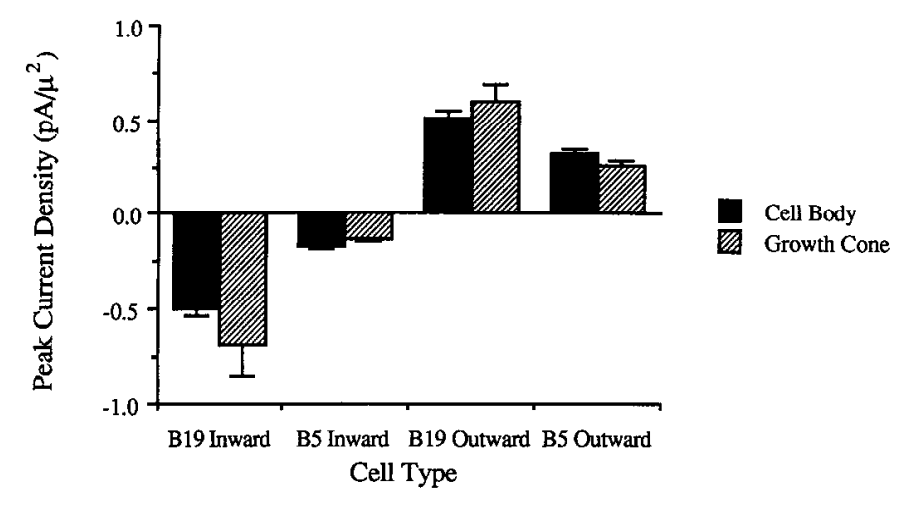

Figure 5. Growth cones and the cell bodies have similar inward and outward current densities. Maximum peak inward and peak outward currents were determined from voltage-clamp records from growth cones and cell bodies of neurons B5 and B19. Neurons and growth cones were held at $-50 \mathrm{mV}$ and given $100 \mathrm{msec}$ steps to $-10 \mathrm{mV}$ for peak inward currents or $+50 \mathrm{mV}$ for peak outward currents. The peak currents were normalized to the estimate of membrane surface area derived from the capacitative current. Values from B19 growth cones $(n=6), \mathrm{B} 19$ cell bodies $(n=12)$, B5 growth cones $(n=13)$, and B5 cell bodies $(n=31)$ were averaged and plotted \pm SEM. Both inward and outward current densities of each type of growth cone were similar to the same measures from the associated cell bodies ( $t$ test: $p>0.15$ ) but different from growth cones of the other neuron type $(t$ test: $p<0.001)$. complementary fractures, which is not possible with our methods.

\section{Discussion}

The correspondence in both electrophysiological and ultrastructural measures between membrane from the cell body and the growth cone is striking. The action potential waveform of the growth cone was indistinguishable from the action potential waveform of the cell body. Although generally used for characterizing individual ionic currents, we have used voltage clamp analysis to demonstrate that the general currents of both growth cone and cell body membranes of the same neuron were characteristic for that neuron. Not only were the time courses and relative magnitudes of the primary inward and outward currents characteristic for the neuron type, the approximate densities of major inward and outward carrying currents were also similar in both the growth cone and the cell body. The ultrastructural studies also found no significant differences in IMP density or size distribution in the growth cone and the cell body. No evidence of organization of the IMPs was seen in the absence of contact with other neurites; the analysis of the IMP reorganization resulting from contact with other neurites from the same neuron, and neurites from other neurons, is being presented in a separate report (R. E. Lee et al., unpublished observations).

Figure 6. Identified regions of a neuron can be easily located in freeze-fracture replicas. A light micrograph of a living neuron B19 is shown at upper left; transmission electron micrographs of freeze-fractured areas correspond to the outlined areas on the light micrograph. Upper right, soma and proximal neurite; middle right, pair of fasiculating neurites; bottom, distal neurites and growth cones. The fractured membrane has been lightly shaded to improve identification. Scale bars: light micrograph, $100 \mu \mathrm{m}$; electron micrograph, $1 \mu \mathrm{m}$. 


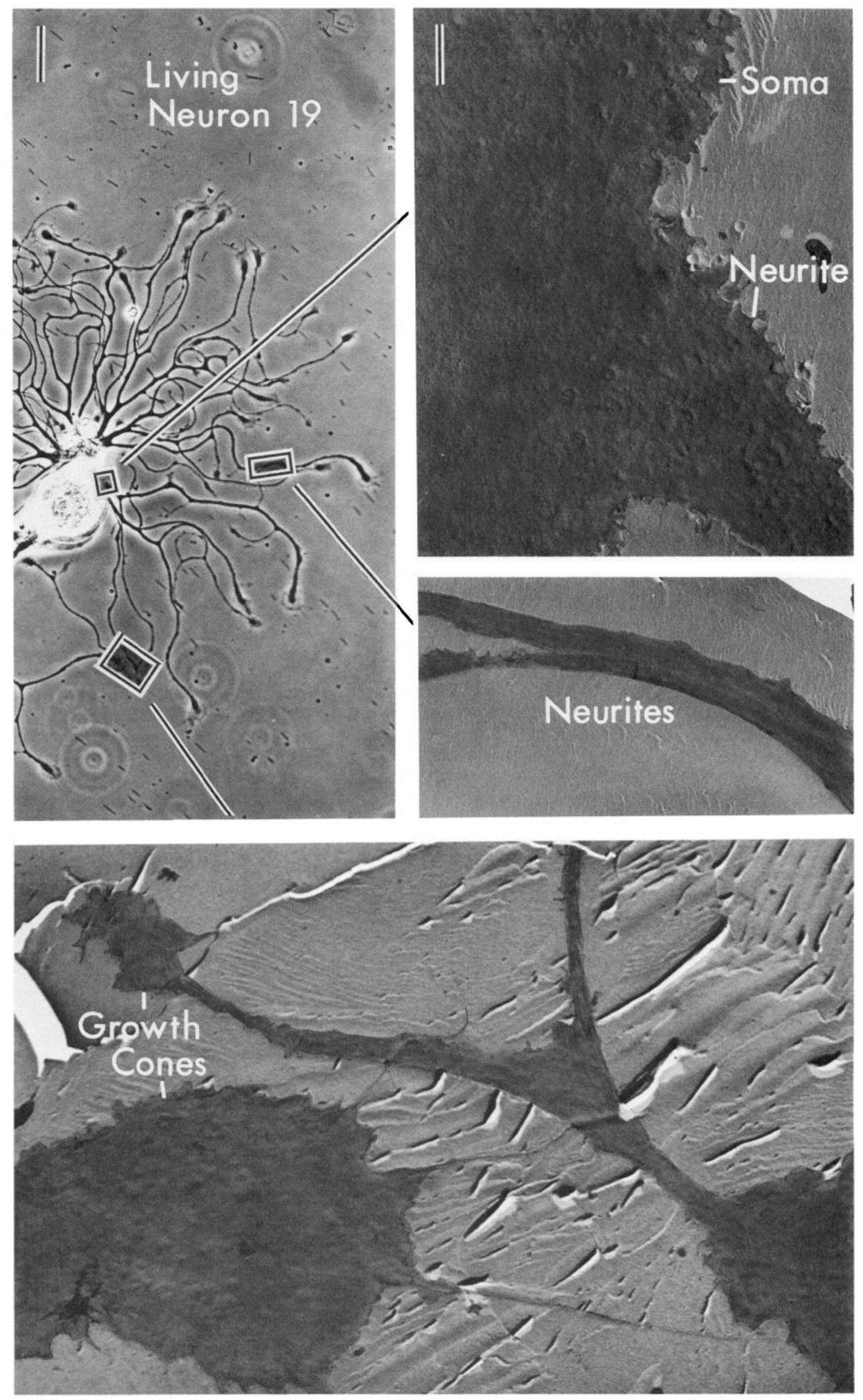


Figure 7. High-magnification freezefracture image of neurite membrane. The 2 fasiculating neurites from Figure 6 (reproduced in inset) are shown at higher magnification. The intramembrane particles (IMPs) in the neurite membrane are clearly visible. Scale bars: inset, $1 \mu \mathrm{m}$; main panel, $0.1 \mu \mathrm{m}$.

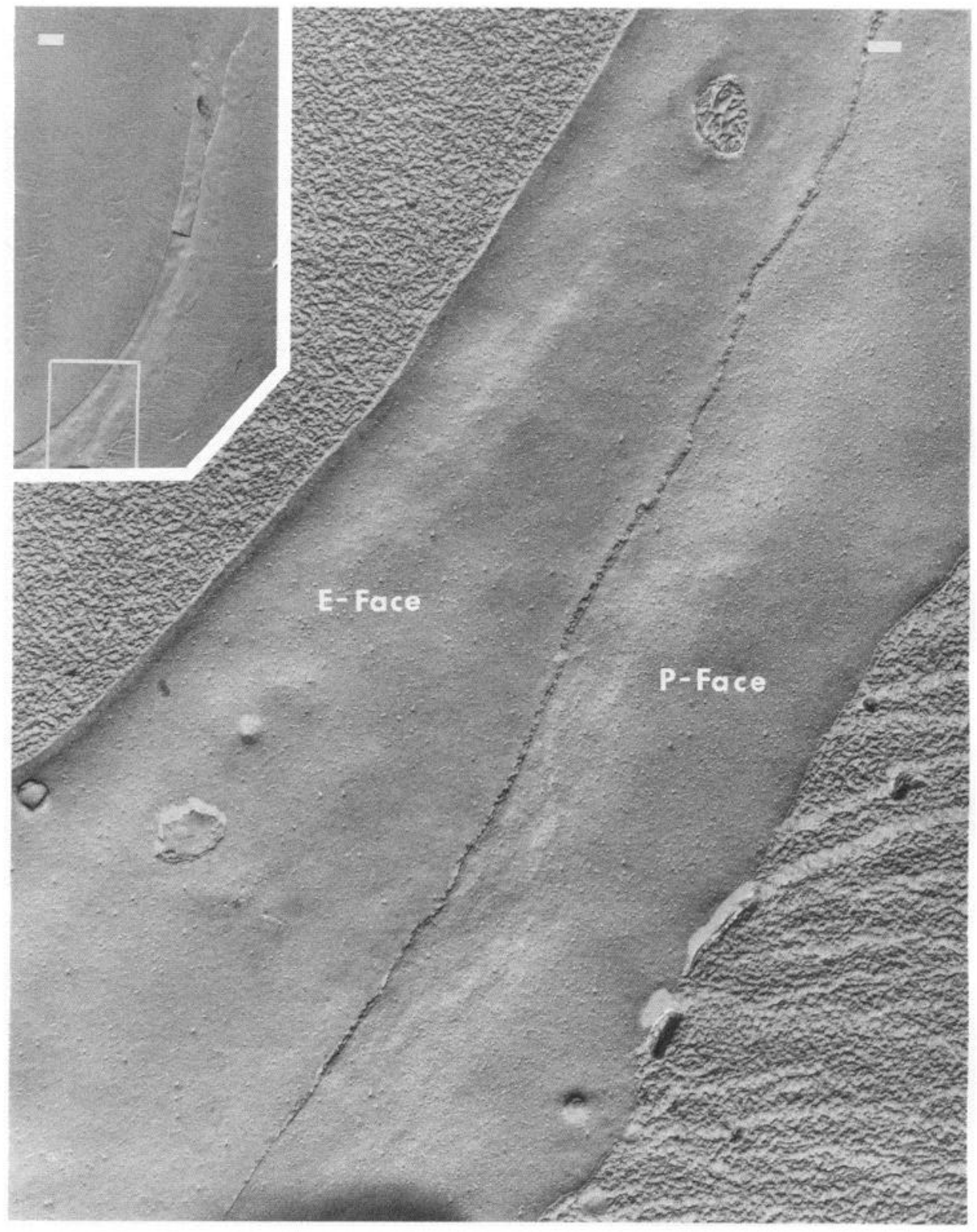

This correspondence between cell body and growth cone membrane properties lends validity to the approach of many neurophysiologists, who, for years, have used recordings from molluscan cell bodies to investigate the properties and plasticity of neurotransmission. Electrophysiological analyses of growth cones in cell culture have now demonstrated the presence of membrane currents similar to those expected from cell body recordings (O'Lague et al., 1985; Belardetti et al., 1986b; Streit and Lux, 1987). In agreement with our results, the A-current was not obvious in growth cones (Belardetti et al., 1986b). Clearly, a dissection of the ionic currents will be necessary to further quantify differences between growth cone and cell body membrane properties. Future experiments must consider that the EGTA in the patch pipette solution used in the present study would have obscured calcium-dependent phenomena, pharmacological manipulations can provide powerful tools for the dissection of specific ionic currents, and finally, the kinetics of specific ionic currents may reveal additional growth cone-cell body differences.

Both electrophysiological and morphological criteria have been used to identify specific neurons as unique individuals in invertebrate nervous systems. The morphology and neurotransmitter responsiveness of some growth cones have also been shown to be characteristically different for growth cones from different neurons. For example, the filopodial number and growth cone surface area of neurons B19 and B5 are significantly different (Haydon et al., 1985). Only a specific subset of neurons responds morphologically to the neurotransmitter serotonin; a different subset responds to dopamine (McCobb et al., 1988). Electrophysiological parameters have been used to identify neurons; they now provide another means of identifying growth cones.

Previous freeze-fracture studies have suggested that a gradient of intramembrane particles exists which is highest towards the 


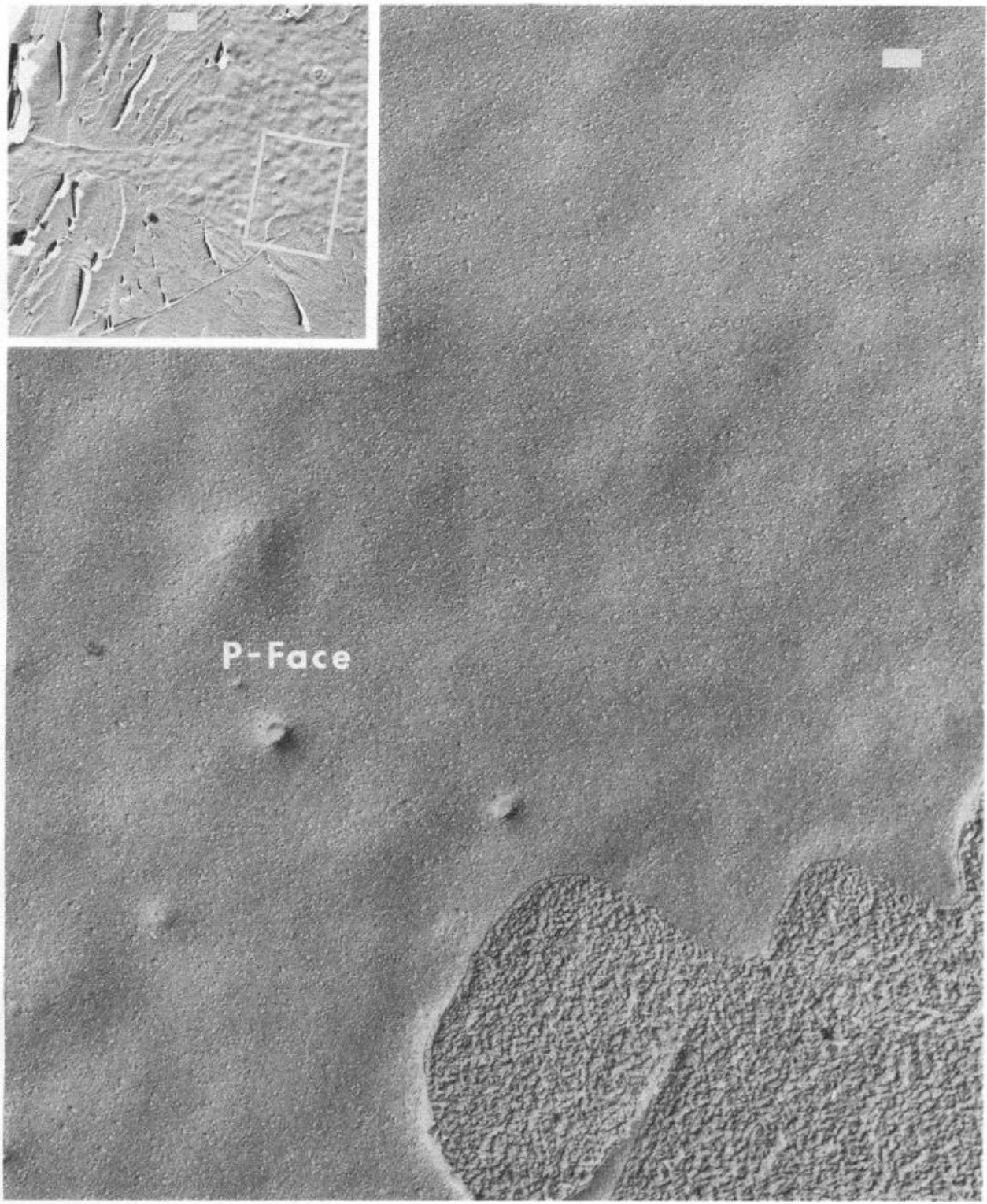

Figure 8. High-magnification freezefracture image of growth cone membrane. A region from one of the growth cones in Figure 6 (growth cone at lower right) is shown at higher magnification. The IMPs in the growth cone membrane are clearly visible. Scale bars: inset, $1 \mu \mathrm{m}$; main panel, $0.1 \mu \mathrm{m}$. cell body and decreases towards the growth cones, which are largely devoid of particles (Pfenninger and Bunge, 1974). One of the implications of these data was that particles were inserted in the cell body and diffused passively along the axon towards the growth cone. Since neurite elongation (and, therefore, general membrane insertion) occurs in the region of the growth cone (Bray, 1970), these results have been somewhat perplexing and conflict with our present observations. There are several interpretations of these differences. Since the growth cones examined in the present experiments are relatively close to the cell body, it is possible that passive diffusion of membrane proteins over short distances could create a relatively uniform membrane. Several observations suggest that this interpretation is unlikely. When growth cones were examined at distances ranging from $50 \mu \mathrm{m}$ to over $500 \mu \mathrm{m}$ from the cell body, no differences were seen between close and distant growth cones. In addition, we did not notice any tendency for IMP density to change in any consistent fashion along even the longest neurites. The one difference that we did notice between the growth cone and the cell body electrophysiological properties (the relative lack of pronounced A-current in the growth cone) was consistent for all growth cones examined. This observation is evidence for selective protein sorting, implying that similarity of growth cone and cell body membrane is due not just to a random diffusion from the cell body membranes into adjacent neurite and growth cone membrane.

In addition, cells grown in culture present at least 4 distinct replica possibilities for examination: both E-face and P-face from both the membrane apposed to the glass coverslip and from the membrane exposed to the culture medium. These 4 replicas would be expected to be different. Studies with reflection contrast interference microscopy have shown that close apposition of the membrane to the surface can affect distribution of specific membrane proteins (Bloch and Geiger, 1980). Specific membrane proteins can also partition differently into the E- and P-faces in different systems. Previous freeze-fracture studies of some invertebrate neurons have shown gap-junction particles to partition into the E-face, while, in vertebrates, gap-junction 

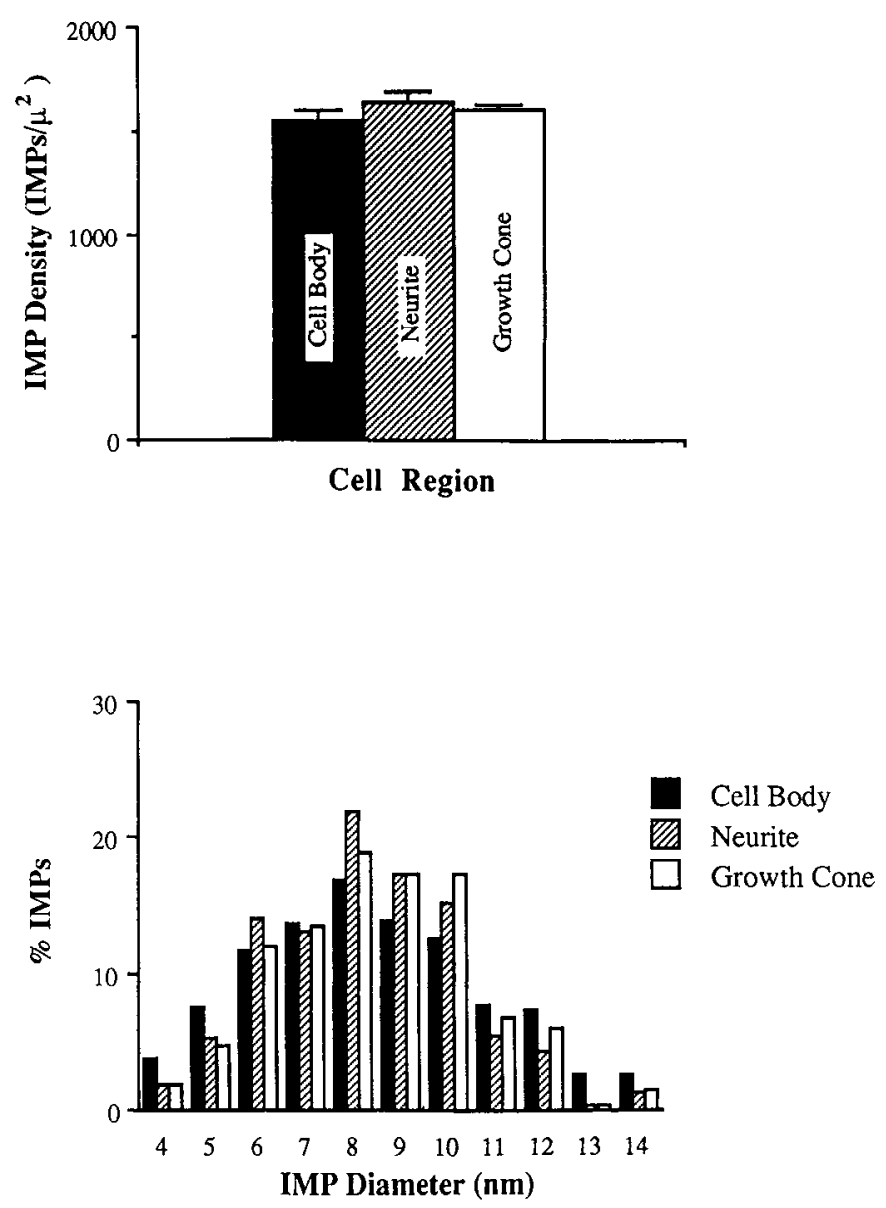

Figure 9. Intramembranous particle (IMP) data from freeze-fracture replicas of identified regions of identified neurons. Buccal neurons B19 were plated onto glass coverslips and fixed during active outgrowth. Representative $1 \mu \mathrm{m}^{2}$ regions from the soma $(n=15)$, neurite $(n=15)$, and growth cone $(n=57)$ were examined. Numbers and diameters of IMPs were determined from photographic prints of replicas at a nominal magnification of $100,000 \times$. The diameters were measured in a direction perpendicular to the shadow with a micrometer loupe. The data from 3 separate neurons were combined in this figure; no significant differences were seen between any of the neurons or between any regions of the neurons. Top, The density of IMPs $/ \mu \mathrm{m}^{2}$ is similar in all regions $(t$ test: $p>0.25)$. Bottom. The distribution of IMP diameters was very similar for all regions of the neuron. The diameters of 1000 IMPs were measured from each region and plotted as the percentage of total IMPs for each diameter catagory. The IMP diameter distributions are not statistically different from each other (Kolgomorov-Smirov test: $p>$ $0.15)$.

particles partition into the P-face (Peracchia, 1973); in molluscs, however, gap-junction particles partition into the P-face (Gilula and Satir, 1971; Berdan et al., 1987; Lee et al., unpublished observations). The primary data presented here are from the P-face of the membrane apposed to the glass coverslip. Some fracture planes did go through the membrane exposed to the medium, however, and represented E-face. Those membranes also showed no differences between growth cone and neurite membrane.

An additional possible resolution to the differences observed in the present result and of those of earlier studies could be derived from the fact that our experiments were performed on regenerating adult neurons in cell culture as a model system. Whilc this possibility remains, it should be recalled, however, that in vivo regenerating neurons of this species have tremendous capacity for appropriate pathfinding and navigation (Murphy and Kater, 1980). Thus, the system employed is one which performs many of the basic functions seen during early development: pathfinding, synapse formation, and the consolidation of synapses. Finally, since it is not possible to resolve the differences between axonal and dendritic growth cones in Helisoma, it remains possible that the axonal growth cones used in earlier studies appear quite different than the neurite growth cones from Helisoma neurons. In any case, the present data clearly indicate that the neuronal growth cone can and does have a disposition of at least some membrane proteins that is strikingly reminiscent of the neuronal soma membrane. These attributes are consistent with the known ability of growth cones to interact with their environment both during development and during regenerative processes.

The similarity in general membrane properties of the cell body and the growth cone membrane leads us to ask whether the electrical properties of the growth cone have any functional significance for regulating the behavior of the growth cone. Electrical activity has been shown to regulate growth cone activity (Cohan and Kater, 1986). Recent evidence strongly supports the hypothesis that the levels of free intracellular calcium are a major factor controlling growth cone activity (Cohan et al., 1987; Mattson and Kater, 1987). The electrical excitability of the growth cone would provide a mechanism for external signals to generate an influx of extracellular calcium. In this regard, the relative lack of a pronounced A-current could be significant. Growth cones receiving a small excitatory stimulus would be more likely to generate action potentials in the absence of an A-current. Another intriguing possibility is that ionic currents might serve as one form of neuronal identification during formation of the nervous system. For example, the amount of current passed across a temporary electrical synapse would be greater for a longer action potential (such as that from a neuron B5) than for a shorter action potential (such as that from a neuron B19). A potential synaptic partner's growth might be stopped by a neuron $\mathrm{B} 5$, stabilizing that synaptic connection and restricting the field of other potential partners; the neuron might continue growing after contact with a neuron B19 and make other synaptic connections, perhaps at the expense of the connection with neuron B19.

The lack of clear, general distinctions between growth cone and cell body membrane properties prompts one to consider a new definition of growth cone. Since growth cone activity is influenced by electrical activity, a functional definition of the growth cone must include those regions that are clectrically close to the growth cone. Since the immediately adjacent neurite is electrically close, it is in position to play a significant role in defining neuronal morphology and must be considered a functional part of the growth cone. In contrast to the functional definition, 3 distinct morphological regions of the neurite/growth cone can be identified: (1) the adjacent neurite with its organized microtubules; (2) the transition zone at the base of the growth cone with its concentration of organelles; and (3) the lampelipodial (and organelle free) region. Mapping the functional regions onto the morphological regions has to be a primary goal for future studies elucidating the mechanisms controlling growth cone behavior. Either loose patch recording or ensemble averaging of single channel recordings will be required to resolve the extent of contribution of the different morphological regions to electrical signals recorded from the growth cone proper. Given their proximity, we will not be surprised to find the bchavior of neuronal growth cones to be significantly biased by the electrical properties of the adjacent neurite membrane. As with any 
structure composed of heterogeneous compartments, the degree of effective space clamp is unknown; therefore, definition of electrical structure may require detailed analysis of the membrane properties at a much finer resolution than currently possible. The specific membrane properties of the different regions of the growth cone/neurite complex, as well as the fine and distant filopodia, may need to be characterized for full resolution of this problem.

While growth cones were first described 100 years ago, detailed studies of growth cone membrane properties are just now beginning (Kater and Letourneau, 1985). We certainly do not have a complete understanding of the full capabilities of growth cones or the mechanisms controlling their behavior. It is obvious that the growth cones of different neurons are different (for instance, in pathfinding; Murphy and Kater, 1980; Raper et al., 1983). The differences we have found in the general electrophysiological properties of growth cones from different neurons may therefore, in part, underlie the diverse capabilities of those growth cones. Such diversity may stem from 3 general levels of organization: (1) growth cone membrane-environment interface; (2) growth cone membrane-cell interior interface; and (3) motile machinery. Given the prominent role for intracellular calcium in growth cone regulation (Cohan et al., 1987), it seems likely that future studies will examine differences in the regulation of calcium levels in growth cones of different identified neurons.

\section{References}

Belardetti, F., S. Schacher, E. R. Kandel, and S. A. Siegelbaum (1986a) The growth cones of Aplysia sensory neurons: Modulation by serotonin of action potential duration and single potassium channel currents. Proc. Natl. Acad. Sci. USA 83: 7094-7098.

Belardetti, F., S. Schacher, and S. A. Siegelbaum (1986b) Action potentials, macroscopic and single channel currents recorded from growth cones of Aplysia neurones in culture. J. Physiol. (Lond.) 374: 289313.

Berdan, R. C., R. R. Shivers, and A. G. M. Bulloch (1987) Chemical synapses, particle arrays, pseudo-gap junctions and gap junctions of neurons and glia in the buccal ganglion of Helisoma. Synapse 1:304323.

Bloch, R. J., and B. Geiger (1980) The localization of acetylcholine receptor clusters in areas of cell-substrate contact in cultures of rat myotubes. Cell 21: 25-35.

Bray, D. (1970) Surface movements during the growth of single explanted neurons. Proc. Natl. Acad. Sci. USA 65: 905-910.

Cohan, C. S., and S. B. Kater (1986) Suppression of neurite elongation and growth cone motility by electrical activity. Science 232 : $1638-$ 1640.

Cuhan, C. S., P. G. Haydon, and S. B. Kater (1985) Single channel activity differs in growing and nongrowing growth cones of isolated identified neurons of Helisoma. J. Neurosci. Res. 13: 285-300.

Cohan, C. S., J. A. Connor, and S. B. Kater (1987) Electrically and chemically mediated increases in intracellular calcium in neuronal growth cones. J. Neurosci. 7: 3588-3599.

Connor, J. A., and C. F. Stevens (1971) Voltage clamp studies of a transient outward membrane current in gastropod neural somata. J. Physiol. (Lond.) 213: 21-30.

Gilula, N. B., and P. Satir (1971) Septate and gap junctions in molluscan gill epithelium. J. Cell Biol. 51: 869-872.

Gundersen, R. W., and J. N. Barrett (1980) Characterization of the turning response of dorsal root neurites toward nerve growth factor. J. Cell Biol. 87: 546-554.

Guthrie, P. B., R. E. Lee, and S. B. Kater (1986) Growth cones of identified neurons: Unique and shared ultrastructural and electrophysiological properties. Soc. Neurosci. Abstr. 12: 370.
Hadley, R. D., R. G. Wong, S. B. Kater, D. L. Barker, and A. G. M. Bullnch (1982) Formation of novel central and peripheral connections between molluscan central neurons in organ cultured ganglia. J. Neurobiol. 13: 217-230.

Haydon, P. G., C. S. Cohan, D. P. McCobb, H. R. Miller, and S. B. Kater (1985) Neuron-specific growth cone properties as seen in identified neurons of Helisoma. J. Neurosci. Res. 13: 135-147.

Haydon, P. G., D. P. McCobb, and S. B. Kater (1987) The regulation of neurite outgrowth, growth cone motility, and electrical synaptogenesis by serotonin. J. Neurobiol. 18: 197-215.

Kater, S. B., and P. Letourneau, eds. (1985) Biology of the Nerve Growth Cone, Liss, New York.

Kater, S. B., and M. P. Mattson (1987) Extrinsic and intrinsic regulators of neurite outgrowth and synaptogenesis in isolated, identified Helisoma neurons in culture. In Cell Culture Approaches to Invertebrate Neuroscience, D. Beadle, G. Lees, and S. B. Kater, eds., Academic, Orlando, FL (in press).

Klein, M., J. S. Camardo, and E. R. Kandel (1982) Serotonin modulates a specific potassium channel in the sensory neurons that show presynaptic facilitation in Aplysia. Proc. Natl. Acad. Sci. USA 79: 5713-5717.

Lankford, K. L., and W. L. Klein (1985) Heterogeneous distribution of lectin binding sites on the cell surfaces of developing central nervous system neurons. Soc. Neurosci. Abstr. 11: 760.

Mattson, M. P., and S. B. Kater (1987) Calcium regulation of growth cone motility and neurite elongation in identified Helisoma neurons. J. Neurosci. 7: 4034-4043.

McCobb, D. P., P. G. Haydon, and S. B. Kater (1988) Dopamine and serotonin inhibition of neurite elongation of different identified neurons. J. Neurosci. Res. 19: 19-26.

Meiri, K. F., K. H. Pfenninger, and M. B. Willard (1986) Growthassociated protein, GAP-43, a peptide that is induced when neurons extend axons, is a component of growth cones and corresponds to pp-46, a major polypeptide of a subcellular fraction enriched in growth cones. Proc. Natl. Acad. Sci. USA 83: 3537-3541.

Murphy, A. D., and S. B. Kater (1980) Differential discrimination of appropriate pathways by regenerating identified neurons in Helisoma. J. Comp. Neurol. 190: 395-403.

O'Lague, P. H., S. L. Huttner, C. A. Vandenberg, K. Morrison-Graham, and R. Horn (1985) Morphological properties and membrane channels of the growth cones induced in PC12 cells by nerve growth factor. J. Neurosci. Res. 13: 301-321.

Pauli, B. U., R. S. Weinstein, L. W. Soble, and J. Alroy (1977) Freezefracture of monolayer cultures. J. Cell Biol. 72: 763-769.

Peracchia, C. (1973) Low resistance junctions in crayfish. II. Structural details and further evidence for intercellular channels by freeze-fracture and negative staining. J. Cell Biol. 57: 66-76.

Pfenninger, K. H., and R. P. Bunge (1974) Freeze-fracturing of nerve growth cones and young fibers: A study of developing plasma membrane. J. Cell Biol. 63: 180-196.

Pfenninger, K. H., and M.-F. Maylie-Pfenninger (1981) Lectin labeling of sprouting neurons. I. Regional distribution of surface glycoconjugates. J. Cell Biol. 89: 536-546.

Rall, W. (1977) Core conductor theory and cable properties of neurons. In Handbook of Physiology, Vol 1, J. M. Brookhart and V. B. Mountcastle, eds., pp. 39-97, American Physiology Society, Bethesda, MD.

Raper, J. A., M. J. Bastiani, and C. S. Goodman (1983) Pathfinding by neuronal growth cones in grasshopper embryos. I. Divergent choices made by the growth cones of sibling neurons. J. Neurosci. 3: 20-30.

Siegelbaum, S. A., J. S. Camardo, and E. R. Kandel (1982) Serotonin and cyclic AMP close single $\mathrm{K}^{+}$channels in Aplysia sensory neurones Nature 299: 413-417.

Skene, J. H. P., R. D. Jacobson, G. J. Snipes, C. B. McGuire, J. J. Norden, and J. A. Freeman (1986) A protein induced during nerve growth (GAP-43) is a major component of growth cone membranes. Science 233: 783-786.

Small, R. K. (1985) Membrane specializations of neuritic growth cones in vivo: A quantitative IMP analysis. J. Neurosci. Res. 13: 39-53.

Streit, J., and H. D. Lux (1987) Voltage dependent calcium currents in PCC12 growth cones and cells during NGF-induced cell growth. Pfluegers Arch 408: 634-641. 